\title{
ANÁLISIS DE LOS SILICOFITOLITOS PRESENTES EN ESPECIES DE las familias Cannabaceae, Moraceae y URticaceae del SE BONAERENSE Y ESTUDIO COMPARATIVO DE LOS CISTOLITOS
}

\author{
MARIANA FERNÁNDEZ HONAINE ${ }^{1,2,4^{*}}$, MARA DE RITO ${ }^{3,4}$ y MARGARITA OSTERRIETH ${ }^{1,2}$
}

\begin{abstract}
Summary: Silicophytolith analyses in species of Cannabaceae, Moraceae and Urticaceae from SE Buenos Aires province and a comparative study of their cystoliths. Numerous families produce silicophytoliths in their tissues, which constitute a relevant taxonomic and palaeobotanical tool. Cystoliths are one of the morphologies produced by dicotyledons, and they are defined as deposits of amorphous calcium carbonate with a silica stalk. This amorphous silica stalk remains on soils and sediments, and constitutes a diagnostic morphology for palaeoenvironmental and archaeological phytolith studies. In this work, the phytoliths of six species of Cannabaceae, Moraceae and Urticaceae, all cystolith producers, were analyzed. Phytoliths were extracted through a calcination technique, and the description of the morphologies and the measurements on cystoliths were carried on through optic and scanning electron microscopes. Statistical tests were applied in order to analyze differences. All the species analyzed produced phytoliths. The first descriptions for three species of Urticaceae and one of Moraceae were shown. Cystoliths, hairs and polyhedrical phytoliths were described, and differentiation was achieved at family level. Celtis spp (Cannabaceae) and Morus alba L. (Moraceae) produce phytoliths different from Urticaceae species, and they present a hair associated, they are thinner and shorter. These features, as well as the morphometric measured variables, could be used for taxa differentiation in fossil phytolith record.
\end{abstract}

Key words: Amorphous silica biomineralization, Morus alba, Celtis spp., Parietaria spp., Urtica urens, SE Pampean region.

Resumen: Numerosas familias producen silicofitolitos en sus tejidos, los cuales constituyen una herramienta de importancia taxonómica y paleobotánica. Una de las morfologías producidas en dicotiledóneas son los cistolitos, definidos como concreciones de carbonato de calcio con un esqueleto silíceo. Este esqueleto silíceo puede quedar preservado en suelos y sedimentos, constituyendo una morfología diagnóstica para estudios fitolíticos paleoambientales y arqueológicos. En este trabajo se analizaron silicofitolitos de seis especies de las familias Cannabaceae, Moraceae y Urticaceae, todas productoras de cistolitos. Los silicofitolitos se extrajeron mediante técnicas de calcinación; se describieron las morfologías y realizaron mediciones a los cistolitos, bajo microscopios óptico y electrónico de barrido. Se aplicaron tests estadísticos para evaluar diferencias. Todas las especies estudiadas producen silicofitolitos, reportándose las primeras descripciones en tres especies de Urticaceae y una de Moraceae. Se describieron cistolitos de variada forma, pelos y fitolitos poliédricos, y fue posible la diferenciación, al menos a nivel de familia. Celtis spp (Cannabaceae) y Morus alba L.(Moraceae) producen cistolitos diferentes a las especies de Urticaceae y presentan una protuberancia o pelo asociado, son más angostos y más cortos. Estas características, así como las variables morfométricas medidas, podrían ser utilizadas para la diferenciación de estos taxa en el registro fitolítico fósil.

Palabras clave: Biomineralizaciones de sílice amorfo, Morus alba, Celtis spp., Parietaria spp., Urtica urens, SE pampeano.

\footnotetext{
${ }^{1}$ Instituto de Geología de Costas y del Cuaternario, Universidad Nacional de Mar del Plata-CIC, CC 722, Correo Central, 7600 Mar del Plata, Argentina.

2 Instituto de Investigaciones Marinas y Costeras (IIMyC), Universidad Nacional de Mar del Plata- Consejo Nacional de Investigaciones Científicas y Técnicas, Mar del Plata, Argentina.

${ }^{3}$ Facultad de Ciencias Agrarias-EEA INTA, Balcarce. Grupo de Estudio de Agroecosistemas y Paisajes Rurales, Ruta 226 $\mathrm{Km} 73,5$, Balcarce, Argentina.

${ }^{4}$ CONICET.

*E-mail: fhonaine@mdp.edu.ar
} 


\section{INTRODUCCIÓN}

Los silicofitolitos son biomineralizaciones de sílice amorfo $\left(\mathrm{SiO}_{2}\right)$ depositados en espacios intra o intercelulares de los tejidos vegetales (Parry \& Smithson, 1964; Bertoldi de Pomar, 1975; Jahren, 1996; Osterrieth, 2004). La formación de estos depósitos de sílice está ampliamente difundida en todos los niveles taxonómicos dentro de las plantas, incluyendo briofitos, pteridofitas, gimnospermas y angiospermas (Piperno, 2006; Mazumdar, 2011; Benvenuto et al., 2013). Los silicofitolitos tienen un alto valor taxonómico, ya que a partir de sus morfologías (que muchas veces copian la forma de las células que los contienen), es posible identificar tejidos y a partir de ellos, las familias y, en algunas ocasiones, los géneros productores (Twiss, 1992; Piperno, 2006; Gomes Coe et al., 2014). Una vez que los órganos vegetales mueren, caen al suelo y se descomponen, los silicofitolitos pasan a formar parte de la fracción mineralógica de los mismos. Dada su alta perdurabilidad en suelos y sedimentos, y su importancia taxonómica, estas biomineralizaciones han sido extensamente utilizadas como indicadoras de las comunidades vegetales del pasado, tanto en estudios paleoambientales y paleobotánicos, como arqueológicos (e.g. Prasad et al., 2011; Mercader et al., 2013; Osterrieth et al., 2014; 2016; Ball et al., 2016).

Las familias Arecaceae, Cyperaceae y Poaceae son las principales productoras de morfologías diagnósticas de silicofitolitos, permitiendo su aplicación como indicadoras de comunidades en el pasado (e.g. Bremond et al., 2005; Strömberg \& Mc Inermy, 2011; Osterrieth et al., 2016). Dentro de las dicotiledóneas, una de las morfologías características a nivel familia o género la constituyen los cistolitos. Desde el punto de vista anatómico, los cistolitos han sido definidos como concreciones de carbonato de calcio formadas sobre excrecencias de la membrana interior de las células (Font Quer, 2001; Metcalfe, 1985). Varios autores han identificado la presencia de un pie o esqueleto silíceo ("silica stalk") sobre el cual se produce el depósito de carbonato de calcio y sin el cual no sería posible el desarrollo del cistolito (Watt et al., 1987; Gal et al., 2012). Este pie o esqueleto silíceo es el que puede quedar preservado en suelos y sedimentos, constituyendo una morfología silicofitolítica de interés para estudios paleoambientales, paleobotánicos y arqueológicos (Osterrieth et al., 2008; Iriarte \& Paz, 2009). Existen varios estudios, tanto anatómicos como fitolíticos, que han identificado y descripto cistolitos en diversos géneros como Celtis y Trema (Dottori, 1976; Bozart, 1992; Wallis, 2003; Iriarte \& Paz, 2009; Watling \& Iriarte, 2013). En particular en nuestro país, se han realizado estudios fitolíticos en ejemplares de Celtis ehrenbergiana (Klotzsch) Liebm. y Celtis occidentalis L. desarrollados en bosques naturales del sudeste bonaerense (Fernández Honaine et al., 2005; De Rito et al., 2015). Sin embargo, si bien los cistolitos son comunes en Cannabaceae, y han sido atribuidos a esta familia cuando son identificados en sedimentos o suelos, es conocido que éstas estructuras silico-cálcicas también están presentes en especies de Moraceae, Urticaceae, entre otras (Metcalfe, 1985; Okazaki et al., 1986; Watt et al., 1987; Gal et al., 2012). Es así que conocer las asociaciones fitolíticas de estos grupos vegetales y analizar si existe diferenciación entre los cistolitos producidos por las distintas familias, resulta relevante a la hora de la interpretación paleoambiental del registro fitolítico fósil. De esta manera, se plantea el presente estudio, cuyos objetivos son: 1) analizar la asociación fitolítica de seis especies pertenecientes a tres familias del orden Rosales, con presencia de cistolitos y desarrolladas en el sudeste bonaerense; 2) realizar un análisis de las formas y medidas de los cistolitos producidos por las especies; 3) analizar comparativamente las morfologías fitolíticas, en particular los cistolitos.

\section{Materiales y Métodos}

Recolección de especies y extracción de silicofitolitos: Se seleccionaron seis especies desarrolladas en distintos ambientes del sudeste bonaerense: Parietaria debilis G. Forst., P. judaica L. (ex Parietaria officinalis auct. non L.), Morus alba L., Urtica urens L., Celtis ehrenbergiana (Klotzsch) Liebm. y Celtis occidentalis L.(Tabla 1). Parte de la toma de muestras fue realizada en el herbario de la Unidad Integrada, Facultad de Ciencias Agrarias-UNMdP/EEA Balcarce, INTA (BAL), y parte en la Reserva Integral Laguna de los Padres y Reserva del Puerto, Partido de Gral. Pueyrredón, Buenos Aires. Si bien las asociaciones fitolíticas de Celtis ehrenbergiana y C. occidentalis 
Tabla 1. Especies seleccionadas para el estudio.

\begin{tabular}{|c|c|c|c|c|}
\hline Familia & Especie & $\begin{array}{l}\text { Herbario y } n^{\circ} \text { de } \\
\text { colección }\end{array}$ & $\begin{array}{l}\text { Fecha y lugar de } \\
\text { recolección }\end{array}$ & Colector \\
\hline Cannabaceae & $\begin{array}{l}\text { Celtis ehrenbergiana } \\
\text { (Klotzsch) Liebm. }\end{array}$ & $\begin{array}{l}\text { Herbario Geoecología de } \\
\text { Ambientes Sedimentarios, } \\
\left.\text { IGCyC, UNMdP ( } n^{\circ} 39\right)\end{array}$ & $\begin{array}{l}\text { 28/3/2004, Laguna de los } \\
\text { Padres, Buenos Aires }\end{array}$ & $\begin{array}{l}\text { Fernández } \\
\text { Honaine, M. }\end{array}$ \\
\hline Cannabaceae & Celtis occidentalis L. & $\begin{array}{l}\text { Herbario Geoecología de } \\
\text { Ambientes Sedimentarios, } \\
\left.\text { IGCyC, UNMdP ( } n^{\circ} 479\right)\end{array}$ & $\begin{array}{l}\text { 13/2/2014, Laguna de los } \\
\text { Padres, Buenos Aires }\end{array}$ & de Rito, M. \\
\hline Moraceae & Morus alba L. & $\begin{array}{l}\text { Herbario BAL, INTA } \\
\text { Balcarce, (BAL2069) }\end{array}$ & $\begin{array}{l}\text { 15/9/96, Parque de la } \\
\text { Compañía Hewlett Packard, } \\
\text { Capital Federal, Bs. As. }\end{array}$ & Azo 1175 \\
\hline Moraceae & Morus alba L. & $\begin{array}{l}\text { Herbario BAL, INTA } \\
\text { Balcarce, (BAL5184) }\end{array}$ & $\begin{array}{l}\text { 14/10/75, Ruta } 226, \\
\text { frente Lag. La Brava, } \\
\text { Balcarce, Bs.As. }\end{array}$ & $\begin{array}{l}\text { herbario viejo } \\
n^{\circ} 229\end{array}$ \\
\hline Moraceae & Morus alba L. & $\begin{array}{l}\text { Herbario BAL, INTA } \\
\text { Balcarce, (CAB401) }\end{array}$ & $\begin{array}{l}\text { 17/10/1983, Ruta } 226, \\
\text { km 62, pie Co. Bachicha, } \\
\text { Balcarce, Bs.As. }\end{array}$ & LM 612 \\
\hline Urticaceae & Urtica urens L. & $\begin{array}{l}\text { Herbario BAL, INTA } \\
\text { Balcarce (BAL4370) }\end{array}$ & $\begin{array}{l}\text { 14/9/71, EEA Balcarce, } \\
\text { Buenos Aires }\end{array}$ & Okada 4091 \\
\hline Urticaceae & Urtica urens L. & $\begin{array}{l}\text { Herbario BAL, INTA } \\
\text { Balcarce (BAL6846) }\end{array}$ & Balcarce, Buenos Aires & $\begin{array}{l}\text { herbario viejo } \\
n^{\circ} 306\end{array}$ \\
\hline Urticaceae & $\begin{array}{l}\text { Parietaria debilis } \\
\text { G. Forst. }\end{array}$ & $\begin{array}{l}\text { Herbario BAL, INTA } \\
\text { Balcarce (BAL4368) }\end{array}$ & $\begin{array}{l}\text { 31/6/66, Sa de la } \\
\text { Ventana, Bs As. }\end{array}$ & Okada 2517 \\
\hline Urticaceae & $\begin{array}{l}\text { Parietaria debilis } \\
\text { G. Forst. }\end{array}$ & $\begin{array}{l}\text { Herbario BAL, INTA } \\
\text { Balcarce (BAL4369) }\end{array}$ & $\begin{array}{l}\text { 20/5/64, Cerro Bachicha, } \\
\text { Balcarce, Bs.As. }\end{array}$ & Okada 1685 \\
\hline Urticaceae & $\begin{array}{l}\text { Parietaria debilis } \\
\text { G. Forst. }\end{array}$ & $\begin{array}{l}\text { Herbario BAL, INTA } \\
\text { Balcarce ( BAL5844) }\end{array}$ & sin datos & $\begin{array}{l}\text { herbario viejo } \\
n^{\circ} 991\end{array}$ \\
\hline Urticaceae & $\begin{array}{l}\text { Parietaria judaica L. } \\
\text { (ex Parietaria officinalis } \\
\text { auct. non L.) }\end{array}$ & $\begin{array}{l}\text { Herbario Geoecología de } \\
\text { Ambientes Sedimentarios, } \\
\left.\text { IGCyC, UNMdP ( } n^{\circ} 350\right)\end{array}$ & $\begin{array}{l}17 / 11 / 2009, \text { Reserva del } \\
\text { Puerto Mar del Plata, Buenos } \\
\text { Aires }\end{array}$ & $\begin{array}{l}\text { Alvarez, } \\
\text { Borrelli, } \\
\text { Fernández } \\
\text { Honaine }\end{array}$ \\
\hline
\end{tabular}

han sido reportadas previamente (Fernández Honaine et al., 2005; De Rito, 2015; De Rito et al., 2015), en el presente trabajo se analizaron en detalle los cistolitos y se compararon las asociaciones fitolíticas con el resto de las especies.

De cada especie se colectaron hojas de dos a tres individuos, las cuales se lavaron en ultrasonido y con agua destilada para eliminar posibles contaminantes. Las muestras se secaron en estufa a $65^{\circ} \mathrm{C}$ por $24 \mathrm{hs}$. Para la extracción de silicofitolitos las muestras se sometieron a la técnica de calcinación de Labouriau (1983) (Fernández Honaine et al., 2005). Esta técnica consiste en una calcinación inicial a $200^{\circ} \mathrm{C}$ por $2 \mathrm{hs}$, una digestión con $\mathrm{HCl} 5 \mathrm{~N}$ y calor para eliminar los carbonatos, y una calcinación final a $800^{\circ} \mathrm{C}$ por $2,5-3$ hs. Como resultado, se obtienen cenizas compuestas exclusivamente por sílice amorfo, ya que los carbonatos y la materia orgánica son eliminados o digeridos por el $\mathrm{HCl}$ y la temperatura, respectivamente.
Descripción de las morfologías fitolíticas: Las cenizas obtenidas por la técnica de Labouriau (1983) fueron montadas en aceite de inmersión para su posterior observación al microscopio óptico Zeiss Axiostar Plus, con 40x de magnificación. Se contaron entre 200- 250 silicofitolitos en cada muestra, se describieron las morfologías, utilizando trabajos de referencia (Madella et al., 2005; Fernández Honaine et al., 2005) y se calcularon las frecuencias relativas de cada una de ellas. Las fotografías fueron tomadas mediante una cámara digital (Canon Powershot G10, Canon Inc., Tokyo, Japón).

Asimismo, para un mejor detalle de las morfologías fitolíticas y de su composición elemental, las cenizas fueron observadas al microscopio electrónico de barrido (JEOL JSM6460 LV; Tokyo, Japan), de la Universidad Nacional de Mar del Plata, y microscopio electrónico de barrido ambiental (ESEM) (Modelo 
FEI ESEM Quanta 200) del LIMF (Universidad Nacional de la Plata). A su vez, para identificar la composición química de los cistolitos, las muestras se analizaron a través de un espectrómetro de rayos $\mathrm{X}$ dispersivo en energías (Modelo: EDAX SDD Apollo 40) del LIMF (Universidad Nacional de la Plata).

Para un análisis en detalle de los cistolitos, se midieron entre 25 y 30 de estas morfologías en cada muestra de cada especie estudiada. Las variables que se midieron en los cistolitos fueron: ancho del tricoma o base (a), largo total del cistolito (b), ancho del pie (si existiere) (c) y diámetro mayor del cistolito (parte globular) (c') (Fig. 1).

Análisis estadísticos: Con el objeto de analizar si es posible la diferenciación de las especies estudiadas, en base a su asociación fitolítica y al tamaño (largo y ancho, variables b y c', respectivamente) de los cistolitos, se realizó un análisis de componentes principales (PCA), donde se utilizó una matriz de correlación (PC-ORD, versión 6, McCune \& Mefford, 2011). Para analizar si existían diferencias en los tamaños (largo y ancho) de los cistolitos se realizaron tests de Kruskal-Wallis y de comparaciones múltiples (Zar, 1984).

\section{Resultados}

Descripción de asociaciones fitoliticas

\section{Celtis spp (Cannabaceae)}

De acuerdo a lo detallado en la sección anterior, las especies de Celtis han sido descriptas por Fernández Honaine et al. (2005) y De Rito (2015). Estos trabajos indican que la asociación fitolítica de Celtis ehrenbergiana está dominada por cistolitos $(88 \%)$, pelos $(5 \%)$, células poliédricas articuladas (4\%) y estomas (Fig. 2 A-C). Mientras que Celtis occidentalis está caracterizado por cistolitos (44\%), pelos silicificados $(37 \%)$ y células epidérmicas silicificadas (18\%) (Fig.2 D-F, 4 I).

\section{Morus alba (Moraceae)}

Las asociación fitolítica de hoja está dominada por cistolitos $(86,4 \%)$, y en menor proporción por pelos $(6,3 \%)$, partes de cistolitos (porción de epidermis solamente $2,7 \%$; sector globular 1,9\%) y fitolitos poliédricos articulados (que derivan de la silicificación de células epidérmicas, 2,1\%) (Fig. 2 G-L, 4 A-C).

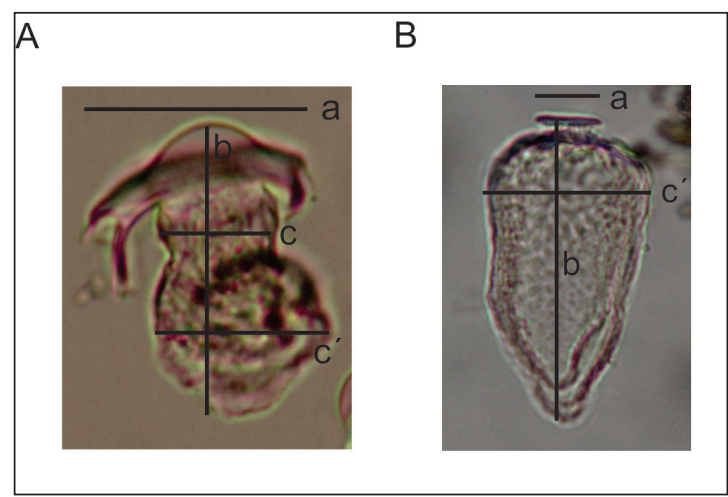

Fig. 1. Fotografías de los dos tipos de cistolitos descriptos en $M$. alba (A) y $U$. urens (B) y las variables medidas en este trabajo. a: ancho del tricoma o base epidérmica, b: largo total del cistolito, c: ancho del pie del cistolito, $c^{\prime}$ : ancho máximo parte globular del cistolito.

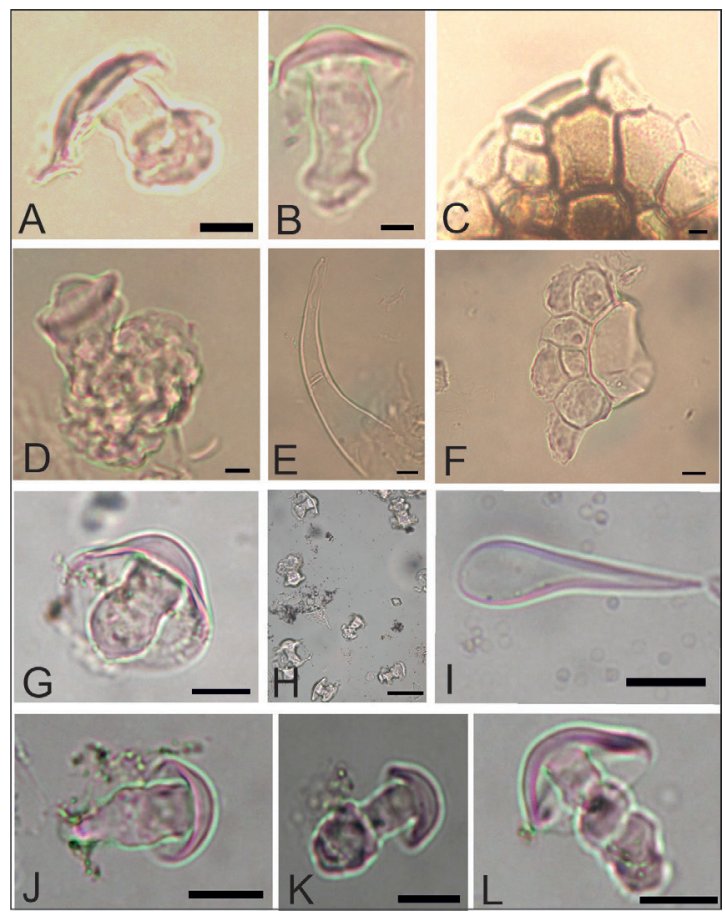

Fig. 2. Silicofitolitos en hoja de Cannabaceae y Moraceae. A-B: Cistolitos de C. ehrenbergiana. C: fitolitos poliédricos derivados de silicificación de células epidérmicas en $C$. ehrenbergiana. $\mathbf{D}$ : Cistolito en C. occidentalis. E: Pelo silicificado en C. occidentalis. F: Fitolito poliédrico en C. occidentalis. G, H, J-L: Cistolitos en M. alba. I: Pelo silicificado en M. alba. Escala $10 \mu \mathrm{m}$. 


\section{Parietaria debilis y $P$. judaica (Urticaceae)}

En ambas especies los pelos silicificados fueron los dominantes, siendo más abundantes en $P$. judaica (71,4\%) que en $P$. debilis $(54,3 \%)$. Los fitolitos globulares, correspondientes al sector globular de los cistolitos, fueron observados en mayor porcentaje en P. debilis $(43,3 \%)$ en relación a $P$. judaica $(24,4 \%)$ (Fig. 3 A-F, 4 G-H). En muy bajos porcentajes (1 y $2 \%$ ) se observaron fitolitos globulares rotos que correspondían al sector globular de los cistolitos.

\section{Urtica urens (Urticaceae)}

Los silicofitolitos más dominantes fueron los globulares alargados, correspondientes a la base globular de los cistolitos $(58,6 \%)$, seguidos por los pelos $(19,2 \%)$ y cistolitos completos con pelos $(9,8 \%)$. Por último, también se observaron fitolitos poliédricos articulados, derivados de la silicificación de células epidérmicas (5,9\%) (Fig. 3 G-I, 4 D-F).

Con el objeto de interpretar si era posible la diferenciación de las especies analizadas, considerando no solo su asociación fitolítica, sino también los tamaños de los cistolitos, se realizó un análisis de componentes principales (PCA) (Fig. 5). Los resultados obtenidos indican que los tres primeros ejes acumulan el $87 \%$ de la variabilidad de los datos $(51 \%$ para el eje $1,21 \%$ para el eje 2 y $15 \%$ para el eje 3 ). Las principales variables que aportan al eje 1 son la abundancia de cistolitos completos, la abundancia de fitolitos globulares, el ancho y el largo de los cistolitos. Es así que este eje separa a Celtis spp. y Morus alba (mayor abundancia de cistolitos completos, en general más angostos y cortos) de Urtica urens y Parietaria spp. (mayor abundancia de fitolitos-cistolitos globulares, más anchos y largos). Al eje 2 aportan mayoritariamente las variables abundancia de fitolitos elongados y fitolitos globulares rotos, presentes en Urtica urens y Parietaria spp., respectivamente. Los fitolitos poliédricos articulados son los que mayoritariamente aportan al eje 3 .

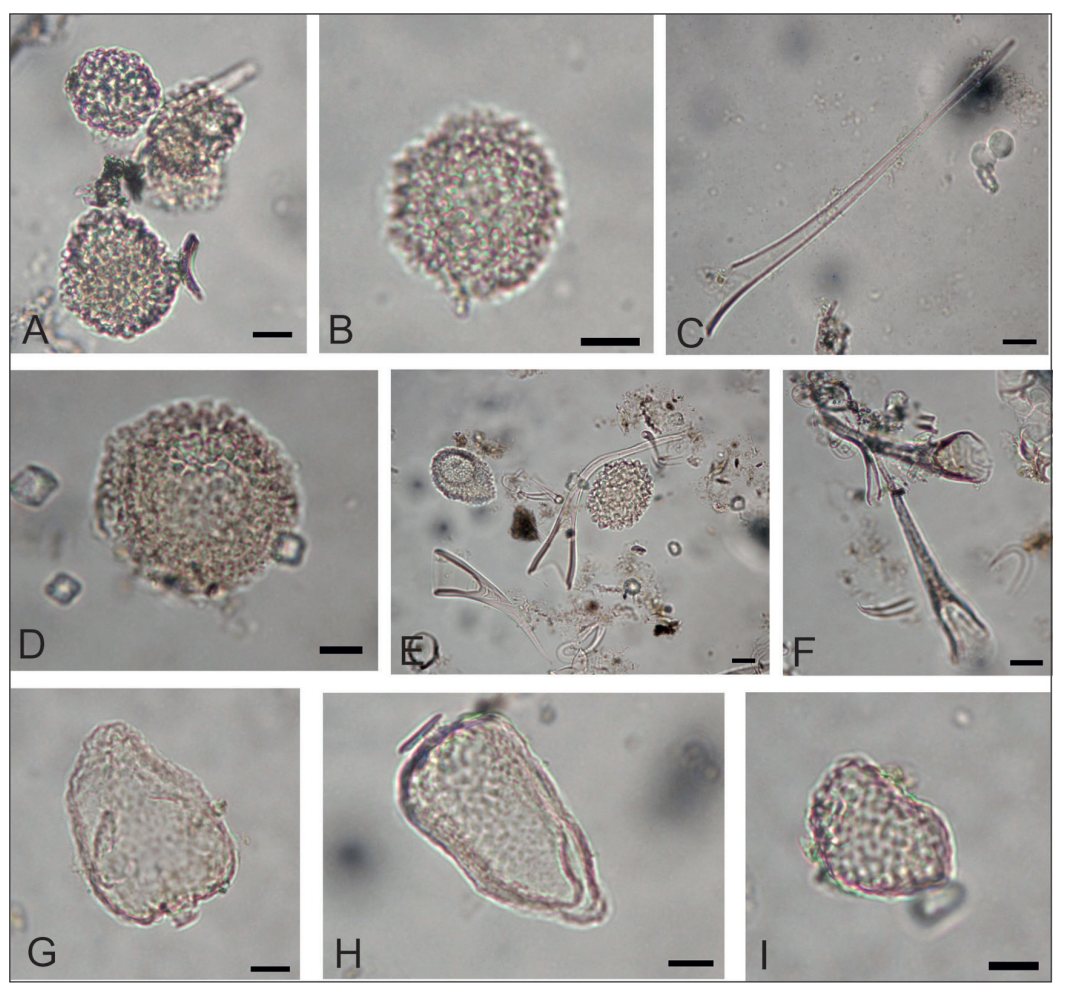

Fig. 3. Silicofitolitos en hoja de Urticaceae. A-B: Cistolitos en P. debilis. C: Pelo en P. debilis. D-E: Cistolitos en P. judaica. F: Pelo en P. judaica. G-I: Cistolitos en U. urens. Escala $10 \mu m$. 

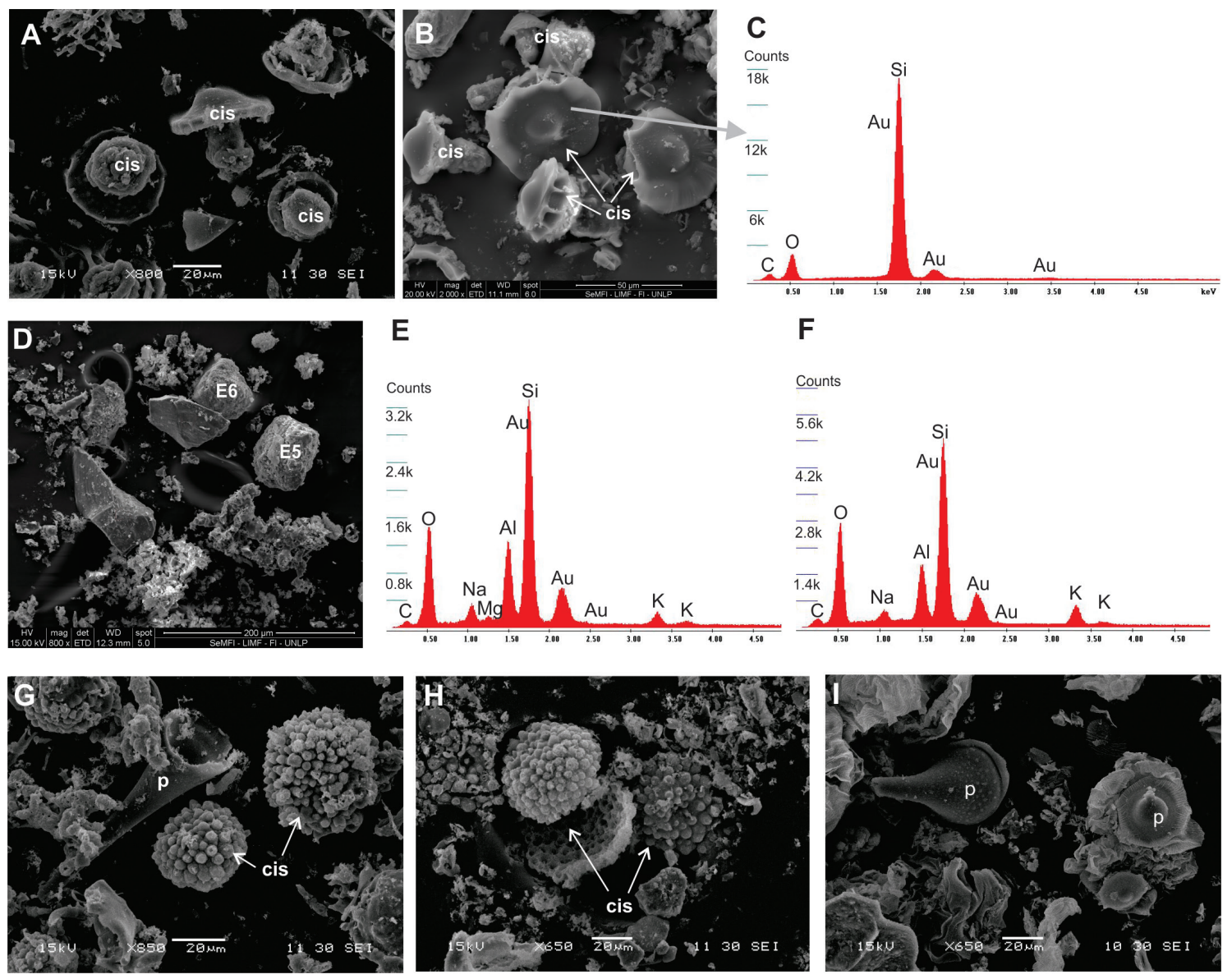

Fig 4. Fotografías obtenidas a partir del microscopio electrónico de barrido de silicofitolitos en hoja, y sus respectivos análisis mediante EDAX. A-B: Cistolitos (cis) en $M$. alba. C: Análisis de EDAX en cistolito fotografiado en $\mathrm{B}$, donde se observa su composición silícea. D: Cistolitos (cis) en U. urens. E: Análisis de EDAX en cistolito fotografiado en $\mathrm{D}$ (E5), donde se observa su composición silícea y de otros elementos. F: Análisis de EDAX en cistolito fotografiado en $D(E 6)$, donde se observa su composición silícea y de otros elementos. G: Cistolitos (cis) y pelos (p) en P. debilis. H: Cistolitos en P. judaica. I: Pelos (p) en C. occidentalis.

\section{Análisis de los cistolitos}

Las observaciones permitieron distinguir dos tipos de cistolitos. El primer tipo está formado por una base de contorno poligonal a elíptico sobre la cual puede desarrollarse un pelo externo, un pie cilíndrico y un cuerpo globoso, de forma ovoide a redondeada. Este tipo de cistolito está presente en Celtis spp. y Morus alba (Fig. 1 A, 2). El segundo tipo de cistolito está representado por cuerpos globosos, en general con una base y pie nulos o apenas desarrollados en relación al cuerpo del cistolito. Está presente en Parietaria spp. y Urtica urens (Figs. 1 B, 3). Es posible diferenciar los cistolitos de estas últimas tres especies por la rugosidad del cuerpo globular. En Parietaria spp. el cuerpo del cistolito presenta una superficie granular, mientras que en Urtica urens el cuerpo es apenas rugoso (Figs. 3, 4 D-H). Los análisis de EDAX permitieron corroborar la composición silícea de los cistolitos, y detectar la presencia de otros elementos, como Al, $\mathrm{K}$ y Na.

En la Tabla 2 se describen las medidas de las diferentes variables analizadas en cada tipo de cistolito. De los análisis estadísticos efectuados surge que existen diferencias significativas en el largo 

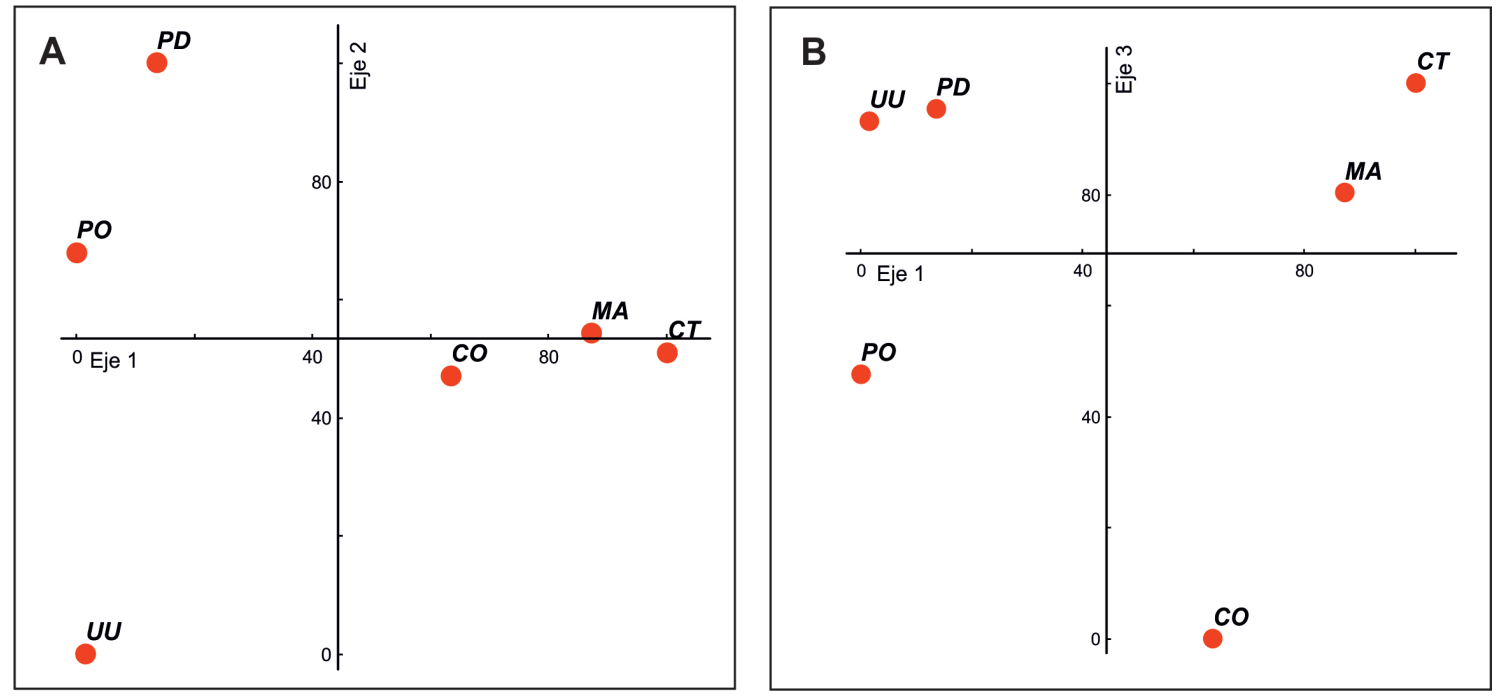

Fig. 5. Análisis de componentes principales, mostrando los tres primeros ejes. CT: Celtis ehrenbergiana, CO: Celtis occidentalis, MA: Morus alba, PD: Parietaria debilis, PO: Parietaria judaica, UU: Urtica urens.

total de los cistolitos entre las especies (KruskalWallis chi-squared $=160,8, \mathrm{p}<0,01)$, siendo mayores los de Urtica urens y $P$. judaica $(54,8$ y $52,4 \mu \mathrm{m}$, respectivamente) y los menores los de $P$. debilis, Morus alba y Celtis ehrenbergiana $(37,1,34,3$ y
$34 \mu \mathrm{m}$, respectivamente). Asimismo, el ancho de los cistolitos es significativamente diferente entre las 6 especies analizadas (Kruskal-Wallis chi-squared $=$ 250,82, $\mathrm{p}<0,01$ ), siendo mayores en Parietaria spp y Urtica urens (Tabla 2).

Tabla 2. Valores medios, mínimos (Mín.) y máximos (Máx.) de las distintas variables medidas en los cistolitos (ver figura 1) (valores en $\mu \mathrm{m}$ ). Letras distintas (considerando una misma variable) indican diferencias significativas $(p<0,05)$. ${ }^{*}$ Indica que solo se pudieron medir en 7 (U. urens) y en 2 ( $P$. judaica) cistolitos, ya que el resto presentaba solo el sector globular.

\begin{tabular}{|c|c|c|c|c|c|c|c|c|c|c|c|c|}
\hline \multirow{3}{*}{ Especie } & \multicolumn{12}{|c|}{ Variables medidas en los cistolitos } \\
\hline & \multicolumn{3}{|c|}{$\begin{array}{l}\text { Ancho de la base del } \\
\text { cistolito (variable a) }\end{array}$} & \multicolumn{3}{|c|}{$\begin{array}{l}\text { Largo total } \\
\text { (variable b) }\end{array}$} & \multicolumn{3}{|c|}{$\begin{array}{l}\text { Ancho del pie } \\
\text { (variable c) }\end{array}$} & \multicolumn{3}{|c|}{$\begin{array}{l}\text { Ancho máximo sector } \\
\text { globular (variable c') }\end{array}$} \\
\hline & Mín. & Media & Máx. & Mín. & Media & Máx. & Mín. & Media & Máx. & Mín. & Media & Máx. \\
\hline Morus alba & 10 & 29.42 & 45 & 20 & $34,29 c$ & 50 & 7.5 & 13.75 & 20 & 7.5 & $18,83 e$ & 35 \\
\hline Urtica urens & 12.5 & $23,21^{*}$ & 32.5 & 20 & $54,80 a$ & 90 & - & - & - & 20 & $41,35 b$ & 67.5 \\
\hline Parietaria debilis & - & - & - & 25 & $37,11 \mathrm{c}$ & 55 & - & - & - & 20 & $34,42 c$ & 52.5 \\
\hline Parietaria judaica & 17.5 & $21,25^{*}$ & 25 & 30 & $52,44 a$ & 80 & - & - & - & 20 & $49,14 a$ & 77.5 \\
\hline Celtis occidentalis & 10 & 29.79 & 75 & 25 & $40,86 b$ & 72.5 & 0 & 14.13 & 25 & 7.5 & $25,39 d$ & 50 \\
\hline Celtis ehrenbergiana & 10 & 27.58 & 50 & 17.5 & $33,97 c$ & 87.5 & 5 & 12.07 & 22.5 & 7.5 & $23,87 d$ & 75 \\
\hline
\end{tabular}




\section{Discusión y Conclusiones}

Todas las especies estudiadas en el presente trabajo producen silicofitolitos, los cuales incluyen cistolitos de variada forma, pelos y fitolitos poliédricos, principalmente. Estas morfologías son el resultado de la silicificación de distintas células del tejido epidérmico (estomas, células epidérmicas en sentido estricto y pelos).

En las especies pertenecientes a la familia Urticaceae (Urtica urens y Parietaria spp.) las morfologías silicofitolíticas dominantes fueron los pelos unicelulares y los cistolitos. Bozarth (1992) describe para Urtica dioica y Parietaria pensylvanica cistolitos similares a los observados en el presente estudio, así como fitolitos derivados de la epidermis (poliédricos y en puzzle). Canti (2003) observa cistolitos en Urtica dioica y Parietaria diffusa semejantes a los aquí estudiados. Collura \& Neumann (2017) describen silicofitolitos en maderas de Urticaceae (Musanga cecropioides), mientras que Bonsen \& ter Welle (1984) mencionan la presencia de cistolitos en tallos y maderas de esta familia. Por su parte, Gal et al. (2012) describen la formación de los cistolitos en Urtica urens bajo distintas condiciones de crecimiento. La familia Moraceae ha sido más ampliamente estudiada desde el punto de vista fitolítico, incluyendo a Morus alba y Ficus spp., entre otras. Kealhoffer \& Piperno (1998) describen en estas especies células epidérmicas silicificadas, bases de pelos, estomas y xilema. Bozarth (1992) describe en Morus rubra cistolitos similares a los observados en el presente trabajo. Tsutsui et al. (2016) analizan la presencia de sílice en forma de papilas en la superficie de hojas de Morus alba, pero no se refieren a los cistolitos. Estas papilas representarían los tricomas bajo los cuales se desarrollan los cistolitos observados en este trabajo. Los antecedentes en silicofitolitos en Cannabaceae son numerosos, e incluyen estudios en hojas, maderas y frutos de especies de Celtis y Trema (Bozarth, 1992; Cowan et al., 1997; Kealhoffer \& Piperno, 1998; Wallis, 2003; Iriarte \& Paz, 2009; Collura \& Neumann, 2017). Todos estos antecedentes, junto a los resultados obtenidos en este trabajo reflejan la alta producción de depósitos de sílice amorfo (ya sea en cistolitos como en células epidérmicas y pelos), en especies de estas familias relacionadas (Moraceae, Urticaceae y Cannabaceae), ahora incluidas dentro del orden Rosales. Asimismo, en otra familia emparentada con las anteriores (familia Cecropiaceae), también se han identificado y descripto idioblastos con $\mathrm{Si}, \mathrm{y}$ en menor porcentaje $\mathrm{Ca}$, sin desarrollo de cistolitos (Setoguchi et al., 1993).

Los cistolitos constituyen una estructura única, compuesta por dos elementos distintos: sílice amorfo y carbonato de calcio (Gal et al., 2012). Originariamente, los cistolitos habían sido definidos como estructuras de carbonato de calcio, depositados en células específicas, denominadas litocistes o litocistos, de origen epidérmico que luego intruyen en el mesófilo (Metcalfe, 1985; Okazaki et al., 1986; Watt et al., 1987; Gal et al., 2012). Sin embargo, estudios más recientes, especialmente el de Gal et al. (2012), confirman la presencia de un esqueleto o pie de sílice amorfo, sin el cual el desarrollo del cistolito no es posible (Watt et al., 1987; Gal et al., 2012). Incluso, estos autores han observado que la falta de disponibilidad de Si impide la formación de ese pie y, como consecuencia, la formación del cistolito (Gal et al., 2012). La estructura de sílice amorfo imita la forma típica de los cistolitos, es decir que aún sin la presencia del depósito calcáreo es posible su identificación como tal, constituyendo una morfología de importancia para los estudios fitolíticos (Bozarth, 1992; Wallis, 2003; Fernández Honaine et al., 2005; Iriarte \& Paz, 2009). Los resultados obtenidos en el presente trabajo reafirman lo observado por los autores antes mencionados, ya que la metodología utilizada elimina los carbonatos presentes en los cistolitos, quedando en las cenizas solo la estructura de sílice amorfo (Labouriau, 1983).

La presencia de cistolitos ha sido registrada y descripta en diversas familias como Acanthaceae, Cannabaceae, Moraceae, Ulmaceae y Urticaceae (Metcalfe, 1985; Setoguchi et al., 1993; Gal et al., 2012). En general, los estudios fitolíticos en estas familias son escasos, y más aún el estudio de los cistolitos (como estructura de sílice amorfo). Los resultados aquí presentados muestran por primera vez las asociaciones fitolíticas de tres especies de Urticaceae y una de Moraceae, todas comunes de la provincia de Buenos Aires. En particular, y comparando con las especies de Celtis analizadas previamente (Fernández Honaine et al., 2005; De Rito, 2015), los resultados indican que si bien las morfologías producidas son cistolitos, pelos y fitolitos poliédricos articulados, es posible la diferenciación, al menos a nivel de familia. Las especies de Celtis spp. (Cannabaceae) y Morus alba 
(Moraceae), producen cistolitos con una estructura diferente a las tres especies de Urticaceae, los cuales en general presentan una protuberancia o pelo asociado y son más angostos y más cortos. Estas características, así como las variables medidas en los cistolitos, podrían ser utilizadas para la diferenciación de los cistolitos en el registro fósil. Los estudios de morfometría aplicado a los silicofitolitos son escasos, pudiendo mencionarse los realizados en silicofitolitos de palmeras de interés comercial (Fenwick et al., 2011), en fitolitos globulares (silicofitolitos característicos de Arecaceae y otras monocotiledóneas nativas de Argentina) (Benvenuto et al., 2015; Patterer, 2014), en ciperáceas del sudeste bonaerense (Fernández Honaine et al., 2009) y en gramíneas (Fahmy, 2008). Este trabajo constituye una contribución a este tipo de estudios, los cuales representan una útil herramienta para la interpretación del registro fitolítico fósil.

\section{Agradecimientos}

A las responsables del herbario BAL INTA, la curadora Ing. Sara Alonso y la Ing. Agr. Ma. Lis Echeverría, quienes permitieron el acceso a las muestras de las especies estudiadas. El trabajo fue financiado por proyectos de la Universidad Nacional de Mar del Plata (EXA 839/17), FONCyT (PICT 1583/13) y CONICET (PIP 0145).

\section{Bibliografía}

BALL T., K. CHANDLER-EZELL, R. DICKAU, N. DUNCAN, T.C. HART, J. IRIARTE, C. LENTFER, A. LOGAN, H. LU, M. MADELLA, D.M. PEARSALL, D.R. PIPERNO, A.M. ROSEN, L. VRYDAGHS, A. WEISSKOP \& J. ZHANG. 2016. Phytoliths as a tool for investigations of agricultural origins and dispersals around the world. J. Archaeol. Sci. 68: 32-45.

BENVENUTO, M.L., M. FERNÁNDEZ HONAINE \& M. OSTERRIETH. 2013. Amorphous silica biomineralizations in Polytrichum strictum Menzies ex Brid. (Bryophyta). J. Bryol. 35: 112-118.

BENVENUTO, M.L., M. FERNANDEZ HONAINE, M. OSTERRIETH \& E. MOREL. 2015. Globular phytoliths differentiation in Arecaceae and other monocotyledons: morphological description for palaeobotanical application. Turk. J. Bot. 39: 341353.
BERTOLDI DE POMAR, H. 1975. Los silicofitolitos: Sinopsis de su conocimiento. Darwiniana 19: 173206.

BONSEN, K.J. \& B.J.H. TER WELLE. 1984. Systematic wood anatomy and affinities of the Urticaceae. Bot. Jahrb. Syst. 105: 49-71.

BOZARTH, S. R. 1992. Classification of opal phytoliths formed in selected dicotyledons native to the Great Plains. In: RAPP, G. \& S. C. MULHOLLAND (eds.), Phytolith Systematics, pp. 193-214. Plenum Press, Nueva York.

BREMOND, L., A. ALEXANDRE, C. HELY \& J. GUIOT. 2005. A phytolith index as a proxy of tree cover density in tropical areas: Calibration with Leaf Area Index along a forest-savanna transect in southeastern Cameroon. Global and Planetary Change 45: 277-293.

CANTI, M.G. 2003. Aspects of the chemical and microscopic characteristics of plant ashes found in archaeological soils. Catena 54: $339-361$.

COLLURA, L.V \& K. NEUMANN. 2017. Wood and bark phytoliths of West African woody plants. Quarter. Int. 434: 142-159.

COWAN, M. R., M. C. GABEL, A. H. JAHREN \& L. L. TIESZEN. 1997. Growth and biomineralization of Celtis occidentalis (Ulmaceae) pericarps. Am. Midl. Nat. 137: 266-273.

DE RITO, M. 2015. Relación entre las asociaciones fitolíticas de especies vegetales y suelos asociados a talares en el sudeste de la provincia de Buenos Aires. Tesis de Grado. Universidad Nacional de Mar del Plata, pp 78.

DE RITO, M., M. FERNANDEZ HONAINE, M. OSTERRIETH \& E. MOREL. 2015. Análisis fitolítico de especies vegetales y suelos asociados a talares en el sudeste bonaerense. Bol. Soc. Argent. Bot. 50 (supl.): 241

DOTTORI, N.M. 1976. Morfología foliar en Celtis tala y Celtis pallida con especial referencia a los domacios. Kurtziana 9: 63-80.

FAHMY, A.G. 2008. Diversity of lobate phytoliths in grass leaves from the Sahel region, West Tropical Africa: Tribe Paniceae. Pl. Syst. Evol. 270: 1-23.

FENWICK, R., C. LENTFER \& M. WEISLER. 2011. Palm reading: a pilot study to discriminate phytoliths of four Arecaceae (Palmae) taxa. $J$. Archaeol Sci. 38: 2190-2199.

FERNANDEZ HONAINE, M., A.F. ZUCOL \& M. OSTERRIETH. 2005. Biomineralizaciones de sílice en Celtis tala (Cannabaceae). Bol. Soc. Argent. Bot. 40 (34): 229-239.

FERNANDEZ HONAINE, M., A.F. ZUCOL \& M. OSTERRIETH. 2009. Phytolith analysis of Cyperaceae from the Pampean Region, Argentina. Aust. J. Bot. 57: 512-523. 
FONT QUER, P. 2001. Diccionario de Botánica. Editorial Península, $2^{\circ}$ Edición, Barcelona, España.

GAL, A., A. HIRSCH, S. SIEGEL, C. LI, B. AICHMAYER, Y. POLITI, P. FRATZL, S. WEINER \& L. ADDADI. 2012. Plant Cystoliths: A Complex Functional Biocomposite of Four Distinct Silica and Amorphous Calcium Carbonate Phases. Chem. Eur. J. 18: 10262-10270.

GOMES COE, H.H., M. OSTERRIETH \& M. FERNÁNDEZ HONAINE. 2014. Phytoliths and their applications. In: GOMES COE, H. \& M. OSTERRIETH (Eds.) Synthesis of some phytolith studies in South America (Brazil and Argentina), pp. 1-26. Series Botanical research and practices. Nova Science Publishers, Inc.

IRIARTE, J. \& E. PAZ. 2009. Phytolith analysis of selected native plants and modern soils from southeastern Uruguay and its implications for paleoenvironmental and archeological reconstruction. Quater. Int. 193: 99-123.

JAHREN, A. H. 1996. How and why do phytoliths form?: Biomineralization. The Phytolitharien 9: $2-10$.

KEALHOFFER, L. \& D.R. PIPERNO. 1998. Opal phytoliths in Southeast Asian flora. Smithsonian Contributions to Botany, (88), pp 39. Smithsonian Institution Press, Washington, D.C.

LABOURIAU, L. G. 1983. Phytolith work in Brazil: a minireview. The Phytolitharien, 2: 6-10.

MADELLA, M., A. ALEXANDRE \& T. BALL. 2005. Internacional code for phytolith nomenclature1.0. Ann. Bot. 96: 253-260.

MAZUMDAR, J. 2011. Phytoliths of pteridophytes. $S$. Afr. J. Bot. 77: 10-19.

MCCUNE, B. \& M. J. MEFFORD. 2011. PC-ORD. Multivariate Analysis of Ecological Data. Version 6.0 MjM Software, Gleneden Beach, Oregon, U.S.A.

MERCADER J., T. BENNETT, C. ESSELMONT, S. SIMPSON \& D. WALDE. 2013. Phytoliths from Middle Stone Age habitats in the Mozambican Rift. J. Hum. Evol. 64: 328-336.

METCALFE, C. R. 1985. Secreted mineral substances. Crystals. In: METCALFE, C.R. \& L. CHALK (eds.) Anatomy of the Dicotyledons Vol II. Wood structure and conclusion of the general introduction, pp: 8297. Ed. Claredon Press, Oxford.

OKAZAKI, M., H. SETOGUCHI, H. AOKI \& S. SUGA. 1986. Application of soft X-ray microradiography to observation of cystoliths in the leaves of various higher plants. Bot. Mat. 99: 281-287.

OSTERRIETH, M., 2004. Biominerales y Biomineralizaciones. In: GARCÍA CALDERÓN N. (ed.) Cristalografía de Suelos, pp. 206- 218. Sociedad Mexicana de Cristalografía. México.
OSTERRIETH, M., M.F. ÁLVAREZ, M. FERNANDEZ HONAINE \& G. ERRA. 2016. Silicophytolith studies in South America and Argentina: scope and limitations for paleoenvironmental reconstruction of the marine isotope stage 3 (MIS3). In: GERMÁN M. GASPARINI, J. RABASSA, C DESCHAMPS \& E. TONNI (eds). Marine Isotope Stage 3 in Southern South America, pp. 321-352. Springer.

OSTERRIETH, M., M. FERNANDEZ HONAINE, N. BORRELLI \& M.F. ALVAREZ. 2014. Silicophytoliths in representative soils of the southeast Pampean Plains, Argentina. In: GOMES COE, H. \& M. OSTERRIETH (eds.) Synthesis of some phytolith studies in South America (Brazil and Argentina), pp 215-241. Series Botanical research and practices, Nova Science Publishers, Inc.

OsterRiETH, M., G. MARTINEZ, M.A. GUTIERREZ \& M.F. ALVAREZ. 2008. Biomorfos de sílice en la secuencia pedosedimentaria del sitio arqueológico Paso Otero 5, Buenos Aires, Argentina. In: KORSTANJE, A. \& P. BABOT (Eds.) Matices interdisciplinarios en estudios fitolíticos y de otros microfósiles, pp. 77-90. BAR International Series S1870, Oxford, Inglaterra.

PARRY, D. W. \& F. SMITHSON. 1964. Types of opaline silica depositions in the leaves of British grasses. Ann. Bot. 28: 169-185.

PATTERER, N.I. 2014. Análisis fitolíticos de las principales especies de palmeras (Arecaceae) presentes en regiones subtropicales de América del Sur. Bol. Soc. Argent. Bot. 49 (4): 491-502.

PIPERNO, D., 2006. Phytoliths. A Comprehensive Guide for Archaeologist and Paleoecologist, pp. 248. Altamira Press.

PRASAD V., C.A.E. STRÖMBERG, A.D. LEACHÉ, B. SAMANT, R. PATNAIK, L. TANG, D.M. MOHABEY, S. GE \& A. SAHNI. 2011. Late Cretaceous origin of the rice tribe provides evidence for early diversification in Poaceae. Nature communications 2: 480.

SETOGUCHI, H., H. TOBE, H. OHBAAND \& $\mathrm{M}$. OKAZAKI. 1993. Silicon-accumulating idioblasts in leaves of Cecropiaceae (Urticales). Plant Res. 106: 327-335.

STRÖMBERG, C. A. \& F.A. MC INERNEY. 2011. The Neogene transition from C3 to C4 grasslands in North America: assemblage analysis of fossil phytoliths. Paleobiology 37: 50-71.

TSUTSUI, O., R. SAKAMOTOA, M. OBAYASHIA, S. YAMAKAWAA, T. HANDAA, D. NISHIOHAMANEB, \& I. MATSUDA. 2016. Light and SEM observation of opal phytoliths in the mulberry leaf. Flora 218: 44-50. 
TWISS, P.C. 1992. Predicted world distribution of C3 and $\mathrm{C} 4$ grass phytoliths. In: RAPP, G. \& S. C. MULHOLLAND (eds.), Phytolith systematics, pp. 113-128. Plenum Press, New York.

WALLIS, L. A. 2003. An overview of leaf phytolith production patterns in selected northwest Australian flora. Rev. Palaeobot. Palynol. 125: 201-248.

WATLING, J. \& J. IRIARTE. 2013. Phytoliths from the coastal savannas of French Guiana. Quater. Int. 287: 162-180
WATT, W. M., C. K. MORRELL, D. L. SMITH \& M. W. STEER. 1987. Cystolith development and structure in Pilea cadieri (Urticaceae). Ann. Bot. 60: 71-84.

ZAR, JH. 1984. Biostatistical Analysis. Prentice-Hall, Englewood Cliffs, NJ, USA.

Recibido el 12 de octubre de 2017, aceptado el 8 de noviembre de 2017. Editora: Georgina M. Del Fueyo. 
